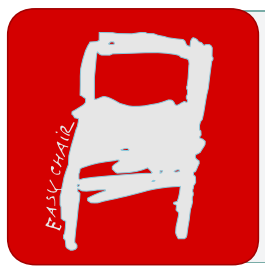

EPiC Series in Health Sciences

Volume 3, 2019, Pages 384-387

CAOS 2019. The 19th Annual Meeting of the International Society for Computer Assisted Orthopaedic Surgery

\title{
Increasing patient empowerment in TKA- recovery via a mobile application: an observational study
}

\author{
Philippe Van Overschelde ${ }^{1}$, Stefaan Van Onsem ${ }^{2}$, Ward Servaes ${ }^{3}$ and Alain \\ Kalmar $^{1}$ \\ ${ }^{1}$ Orthopaedic department, AZ Maria Middelares, Gent, Belgium \\ ${ }^{2}$ Orthopaedic department, University Hospital, Gent, Belgium \\ ${ }^{3}$ moveUP cvba, Belgium \\ Stefaan.vo@gmail.com
}

\begin{abstract}
During the last few years, total knee arthroplasty has evolved from basic surgery to restore basic function, to a fast-track procedure to relief pain and get the patients back to a high level of activity.. However, the way physiotherapy is performed, remained the same. This paper presents the results of 185 patients that used a mobile health application to track their recovery. Adherence and clinical results were evaluated and reported. Our study demonstrated an excellent patient acceptance and compliance of the outpatient autonomous recovery app. Patient satisfaction was outstanding, and functional recovery was securely within conventional range.
\end{abstract}

\section{Introduction}

In the last half-century, total knee arthroplasty (TKA) has evolved tremendously to routine surgery with faster recovery, optimized pain-control, immediate mobilisation, shorter hospital stay and predictable outcome $^{1}$. Despite, supervised physiotherapy, either at an outpatient facility or an inpatient rehabilitation centre, has remained the gold standard ${ }^{2}$. As an alternative to conventional rehabilitation, a dedicated program of individualised telerehabilitation may prove effective to improve functional outcome and patient satisfaction ${ }^{2,3}$. In anticipation of a randomized outcome study in TKA patients, we performed an observational study to assess the patient acceptance and compliance using a recovery app for remote patient surveillance and care. 


\section{Patients and methods}

More than 200 patients used the proposed mobile application for their recovery after TKA surgery between June 2017 and October 2018. This application monitors the number of daily steps via activity tracker, evaluates pain levels (day and night), sleep quality, inflammation, stiffness, medication and

type of activity performed, as reported by the patients. Data was captured 1 week before their surgery (in order to have a baseline) and from discharge on (mean length of stay: 3 days). Through objective and subjective feedback, the app accommodates the rehabilitation program and provides personalised feedback. In addition, the physiotherapist is notified in case of anomalies through a smart filtering system. Every patient was evaluated on usability and digital acceptance.

\section{Results}

This study depicts the results of the first 185 patients that used the aforementioned application until the end of their rehabilitation. Usability-scores and adherence of the application was found to be very high. Mean adherence (number of daily questionnaires completed on total number of days until the end of rehab) $84 \%$ (SD 17\%)(Median 90\%). 78\% percent of the patients were promotors of the system, $8 \%$ detractors and $14 \%$ undecided. (see figure 1)

The following clinical results were found: Mean age 64 (range 30-90), mean BMI $28 \mathrm{~kg} / \mathrm{m}^{2}$, equal gender distribution, mean opioid stop after 3 days, mean stop paracetamol after 24 days, the knee society satisfaction subscore increased from 18 preoperatively to 26 at 6 weeks, 28 at three months 34 at 6 months (see figure 1). At 6 weeks only $6 \%$ reports pain during the night and $8 \%$ during the day. At 3 months none of the patients reported pain during the night or during the day. 

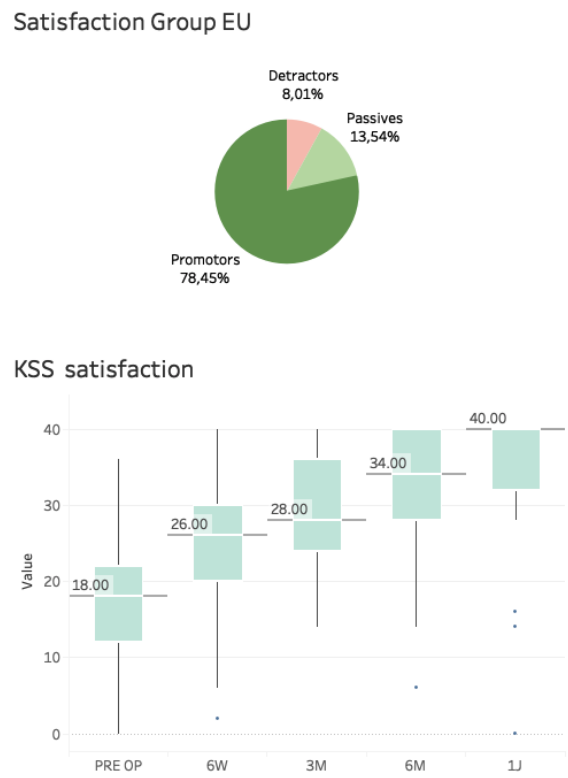

No own tablet or smartphone

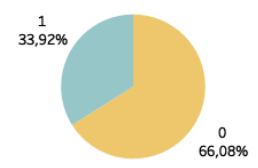

Topics

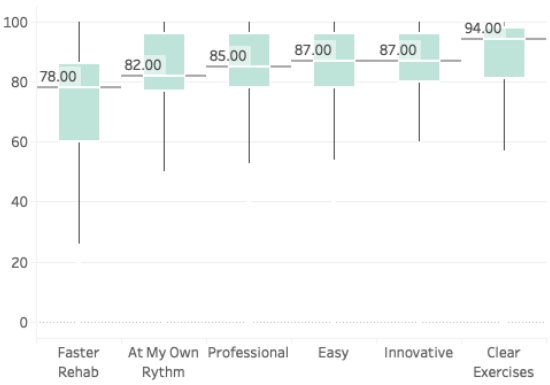

figure 1: left upper corner: amount of promotors, detractors and passives in the EU patient population.

Right upper corner: \% of people owning a tablet or smartphone $(0=n o, 1=y$ es $)$, left lower corner, boxplots of KSS satisfaction (max score 40), preoperative, 6 weeks, 3 months, 6 months and 1 year postoperative. Right

lower corner, description of the application by the users

\section{Discussion}

Historically, supervised physiotherapy either at an outpatient facility or an inpatients rehabilitation centre, has been widely accepted as standard of care after TKA surgery ${ }^{2}$. However, there is limited literature to support this assumption ${ }^{4}$. Our study demonstrated an excellent patient acceptance and compliance of the outpatient autonomous recovery app. Patient satisfaction was outstanding, and functional recovery was securely within conventional range 5 . Furthermore, the majority of the patients would recommend the use of this application, even though some did not have a tablet or smartphone before the use of this application.

\section{Conclusion}

This observational study confirms our hypothesis that reliable compliance is reached, and the technology may permit optimisation of the rehabilitation process. A randomized controlled trial comparing conventional and the use of the tele-rehabilitation app will evaluate the effect on functional outcome and patient satisfaction. Further research will evaluate if these advancements in rehabilitation, by permitting close patient surveillance and monitoring in an outpatient setting, may provide better outcomes and significant cost savings. 


\section{References}

1. Husted H. Fast-track hip and knee arthroplasty: Clinical and organizational aspects. Acta Orthop H. Husted, Dept. of Orthopaedic Surgery 333, University Hospital of Hvidovre, Copenhagen, DK-2650 Hvidovre, Denmark, 2012;83(SUPPL.346):1-39.

2. Fleischman AN, Crizer MP, Tarabichi M, Smith S, Rothman RH, Lonner JH, et al. 2018 John N. Insall Award: Recovery of Knee Flexion With Unsupervised Home Exercise Is Not Inferior to Outpatient Physical Therapy After TKA: A Randomized Trial. Clin Orthop Relat Res 2019;477(1):60-69.

3. Taunton MJ. What' $\mathrm{s}$ New in Adult Reconstructive Knee Surgery. J Bone Jt Surg 2019;101:103-111.

4. Artz N, Dixon S, Wylde V, Beswick A, Blom A, Gooberman-Hill R. Physiotherapy Provision Following Discharge after Total Hip and Total Knee Replacement: A Survey of Current Practice at High-Volume NHS Hospitals in England and Wales. Musculoskeletal Care 2013;

5. Van Onsem S, Verstraete M, Dhont S, Zwaenepoel B, Van Der Straeten C, Victor J. Improved walking distance and range of motion predict patient satisfaction after TKA. Knee Surgery, Sport Traumatol Arthrosc 2018;1-8. 